\title{
Peculiarity of the oscillation stratification in sunspot penumbrae
}

\author{
D.Y. Kolobov, A.A. Chelpanov and N.I. Kobanov \\ Institute of Solar-Terrestrial Physics \\ of Siberian Branch of Russian Academy of Sciences, Irkutsk, Russia \\ email: kolobov@iszf.irk.ru \\ [This article was firstly published in Solar Physics DOI]
}

\begin{abstract}
Spatial distributions of the dominant oscillation frequency obtained for four sunspots show a feature shared by all the analysed levels of the solar atmosphere in these sunspots. This feature located in the inner penumbrae indicates that this region has favourable conditions for $2.5-4 \mathrm{mHz}$ oscillation propagation. This agrees with the fact that the spectral composition of the oscillations at three atmospheric heights (Fe I $6173 \AA, 1700 \AA$, and He II $304 \AA$ ) in this region are similar. There have been previous evidence of particular similarities along height of photospheric magnetic field strength, line-of-sight velocity, and temperature profile in the inner penumbra, where the internal boundary of the Evershed flow is located. The finding of the same dominant oscillation frequency at a range of altitudes from the chromosphere up to the transition region extends the height range, suggesting similarities in physical conditions.
\end{abstract}

\section{Introduction}

Sunspots have long been an important object for the study of oscillations and waves in the solar atmosphere. Sunspots provide a diverse range of interactions between the solar magnetic field and matter. The umbra, where the vertical magnetic field prevails, shows signatures of downward motion and weak five-minute oscillations of the whole umbra at the photospheric level (Lites, 1988; Kobanov, 1990). In the chromosphere, strong three-minute oscillations dominate in the umbra; these oscillations at first were considered as standing acoustic waves (Lites, 1988; Georgakilas, Christopoulou, and 
Koutchmy, 2000). Later, these waves were shown to be moving upward (Rouppe van der Voort et al., 2003); also they were shown not to be a simple continuation of the running penumbral waves (RPW) in the horizontal direction (Kobanov, Kolobov, and Makarchik, 2006). New observations with ever-increasing spatial and temporal resolution capabilities reveal many new facts about the sunspot's fine structure (Jess et al., 2015; Khomenko and Collados, 2015; Yuan et al., 2014; Sych and Nakariakov, 2014; Sych, 2015).

Penumbra is a complex part of a sunspot, whose understanding and modelling are largely complicated by horizontal - or rather a mixture of differently inclined - flows, steep temperature gradient, and rapid change of the magnetic field strength towards the outer boundary. Further complexity arises due to the inhomogeneity in the azimuthal direction: the bright and dark filaments are associated with different physical parameters. The magnetic field inclination has been shown to be larger in the dark filaments forming the so-called uncombed penumbra (Title et al., 1993; Solanki and Montavon, 1993; Bellot Rubio, 2003). Recently, with the help of highresolution instruments, two components of the magnetic field were observed in a sunspot penumbra. Both components have an inclination close to $40^{\circ}$ in the inner penumbra, and in the outer penumbra the dark-filament field grow horizontal, while the bright-filament field only reaches $60^{\circ}$ inclination (Langhans et al., 2005).

It is necessary to analyse the oscillations in the penumbra to form a comprehensive picture of the waves propagating in sunspots. A wide range of frequencies is observed in sunspot penumbrae: Lites (1988); Brisken and Zirin (1997); Zirin and Stein (1972) registered oscillations in intensity and Doppler velocity signals. Different frequencies tend to appear at different regions of the penumbra: typically, the longer the period, the farther it is observed from the sunspot centre. Sigwarth and Mattig (1997) noted that this change in the frequency is more pronounced in the chromosphere than in the photosphere. Such a distribution is explained by the increase in the magnetic field inclination closer to the boundaries of a sunspot, and thus the decrease in the cut-off frequency (Reznikova and Shibasaki, 2012; Reznikova et al., 2012; Kobanov, Chelpanov, and Kolobov, 2013).

In a sunspot there is a phenomenon called running penumbral waves (RPW) - observed increase in brightness travelling outwards the penumbra (Beckers and Tallant, 1969; Giovanelli, 1972; Zirin and Stein, 1972). These waves span a range of frequencies from 1 to $4 \mathrm{mHz}$ (Lites, 1988). Lites (1988); Brisken and Zirin (1997); Jess et al. (2013) showed that the periods 
of the waves increase closer to the boundaries of a sunspot. RPWs were found at the photospheric heights as well (Musman, Nye, and Thomas, 1976; Löhner-Böttcher and Bello González, 2015). Naturally, the question was raised on the origin of RPWs: two concepts were proposed. The first one is that RPWs are real waves propagating horizontally across the penumbra (Alissandrakis, Georgakilas, and Dialetis, 1992; Tsiropoula, Alissandrakis, and Mein, 2000; Tziotziou et al., 2004, 2006); and the second concept implies them to be an apparent effect - a result of waves rising to the surface along inclined magnetic tubes, thus appearing first at the inner penumbra and then farther from the sunspot centre (Rouppe van der Voort et al., 2003; Bogdan and Judge, 2006; Kobanov, Kolobov, and Makarchik, 2006; Bloomfield, Lagg, and Solanki, 2007; Kobanov, Kolobov, and Chupin, 2008; Cho et al., 2015). The same effect is also deemed to be responsible for umbral flashes; and indeed, recently, researchers tend to consider umbral flashes and RPWs as manifestations of one phenomenon (Madsen, Tian, and DeLuca, 2015). This second explanation raises a consequent question: what is the origin of the waves responsible for the observed effect? A number of authors come to the conclusion that these waves are slow magnetoacoustic modes resulting from photospheric p-mode oscillations (Bloomfield, Lagg, and Solanki, 2007; Madsen, Tian, and DeLuca, 2015) or a broadband energy deposition process, e.g. granulation motions (Botha et al., 2011). Jess et al. (2013) studied the influence of the magnetic field inclination on the RPW. They concluded that the increase in the inclination leads to the increase in the dominant periodicity due to the dropping of the cut-off frequency. However, due to the complicated dynamical properties, reliable estimations of the RPW parameters are difficult to carry out.

The important feature of the oscillations discussed above is their relation to the Evershed flow. Kobanov and Makarchik (2004) found three ranges of oscillations that most likely connect the direct Evershed flow and the inverse - the so-called St. John's - flow. The 20-35-minute oscillations have the most consistent phase difference between the photospheric and chromospheric heights.

In our previous works (Kobanov, Kolobov, and Chelpanov, 2015; Kolobov et al., 2015), we revealed that the dominant frequency spatial distribution shows a peculiarity in sunspots' penumbra. We constructed plots showing the dominant frequencies averaged in the azimuthal direction as a function of the distance to sunspot's barycentre, and these plots converged in the inner penumbra. This feature corresponds to the $3-4 \mathrm{mHz}$ frequency range, which 
Table 1. Data series used in the analysis

\begin{tabular}{crrccc}
\hline No. & NOAA & Date & $\begin{array}{c}\text { Disc } \\
\text { location }\end{array}$ & $\begin{array}{c}\mathbf{T}_{\text {start }} \text { (UTC) } \\
\text { hh:mm }\end{array}$ & $\begin{array}{c}\mathbf{T}_{\text {end }} \text { (UTC) } \\
\text { hh:mm }\end{array}$ \\
\hline & & & & \\
1 & 11311 & 6 Oct 2011 & $11^{\circ} \mathrm{S} 08^{\circ} \mathrm{E}$ & $03: 21$ & $04: 45$ \\
2 & 11479 & 16 May 2012 & $15^{\circ} \mathrm{N} 11^{\circ} \mathrm{E}$ & $00: 07$ & $07: 59$ \\
3 & 11711 & 6 Apr 2013 & $17^{\circ} \mathrm{S} 03^{\circ} \mathrm{W}$ & $00: 00$ & $04: 20$ \\
4 & 12149 & 27 Aug 2014 & $10^{\circ} \mathrm{N} 02^{\circ} \mathrm{W}$ & $06: 00$ & $10: 00$ \\
\hline
\end{tabular}

indicates that five-minute oscillations dominate above the inner penumbra at all the heights from the photosphere to the transition region. One can assume that in this ring-shaped region above the inner penumbra, favourable physical conditions for five-minute wave propagation exist.

\section{Methods}

For this study we used full-disk narrow-band images provided by the Atmospheric Imaging Assembly (AIA: Lemen et al., 2012) onboard the Solar Dynamics Observatory (SDO). Of all the spectral bands used in the observations we chose four: $1700 \AA$, He II $304 \AA$, Fe IX $171 \AA$ obtained by AIA, and Fe I $6173 \AA$ obtained by Helioseismic and Magnetic Imager (HMI: Scherrer et al., 2012). These lines cover the height range from the deep photosphere to the corona.

The AIA data have time resolution 12 and $24 \mathrm{~s}$, depending on the spectral band. Each pixel of these data corresponds to $0.6^{\prime \prime}$. The HMI data have a $45 \mathrm{~s}$ time resolution and $0.5^{\prime \prime}$ detector pixel size. Fe I $6173 \AA$ line is formed in the photosphere at a height of $200 \mathrm{~km}$ (Beckers and Tallant, 1969). Also, the Doppler velocity maps and magnetograms are available in this line.

In the analysis we used four sunspots in NOAA 11311, 11479, 11711, and 12149 (Table 1). In all four cases the spots had roughly regular round shapes and located close to the disk centre, and thus the effects of the foreshortening and asymmetry were minimal. The data series lengths ranged from 4 to 8 hours, and no flares were registered during these observations.

After removing trends from the time series, the power spectra were calculated by Fast Fourier Transform (FFT) using the standard routine of 
the Interactive Data Language (IDL). Based on these spectra, we constructed the dominant frequency distributions within the analysed regions. To this end, the spectrum from each pixel was smoothed using convolution with a $1 \mathrm{mHz}$ window, and the maximum frequency value of the resulting function was considered as the dominant frequency within the given pixel.

\section{Results and Discussion}

Oscillations of different frequencies are observed in sunspots, and within a sunspot they are distributed non-uniformly. First, different frequency bands occupy different regions of a sunspot. High-frequency oscillations tend to be located within the umbra boundaries, while lower frequencies form circles, whose radii increase with the decreasing frequency. Such distributions are believed to be related to the magnetic field configuration, namely, magnetic field inclination angle: the location of high frequencies coincides with that of the vertical magnetic field, while low frequencies are located at the high-inclination outer penumbra (Reznikova and Shibasaki, 2012; Kobanov, Chelpanov, and Kolobov, 2013). Second, the circles of oscillation distributions grow with the height. Such a pattern, again, is deemed to be related to the magnetic field configuration: waves propagating along magnetic field lines inclined from the spot centre approach the spot's outer boundaries at each consecutive height level (Kobanov, Chelpanov, and Kolobov, 2013).

Figure 1 shows spatial distributions of the dominant frequencies based on FFT power spectra. The distributions are reconstructed from the SDO data. They follow the two aforementioned features of the oscillation behaviour in sunspots. Based on these distributions we plotted profiles of dominant frequencies as functions of distance from a sunspot centre $r$ presented in Figure 2. These profiles were plotted for three height levels - from the photosphere to the transition region - in the four sunspots.

These plots behave as we expected-high-frequency range in the centre and gradual decrease towards the sunspot boundaries. The interesting feature in all the studied sunspots that caught our attention is the convergence of all the profiles in the inner penumbrae (see Figure 2). In case of NOAA 11711 the profiles intersect in this region. Figure 3 shows intensity oscillation power spectra azimuthally averaged over the region marked in Figure 2. The panels for NOAA 12149 show that in the transition region 

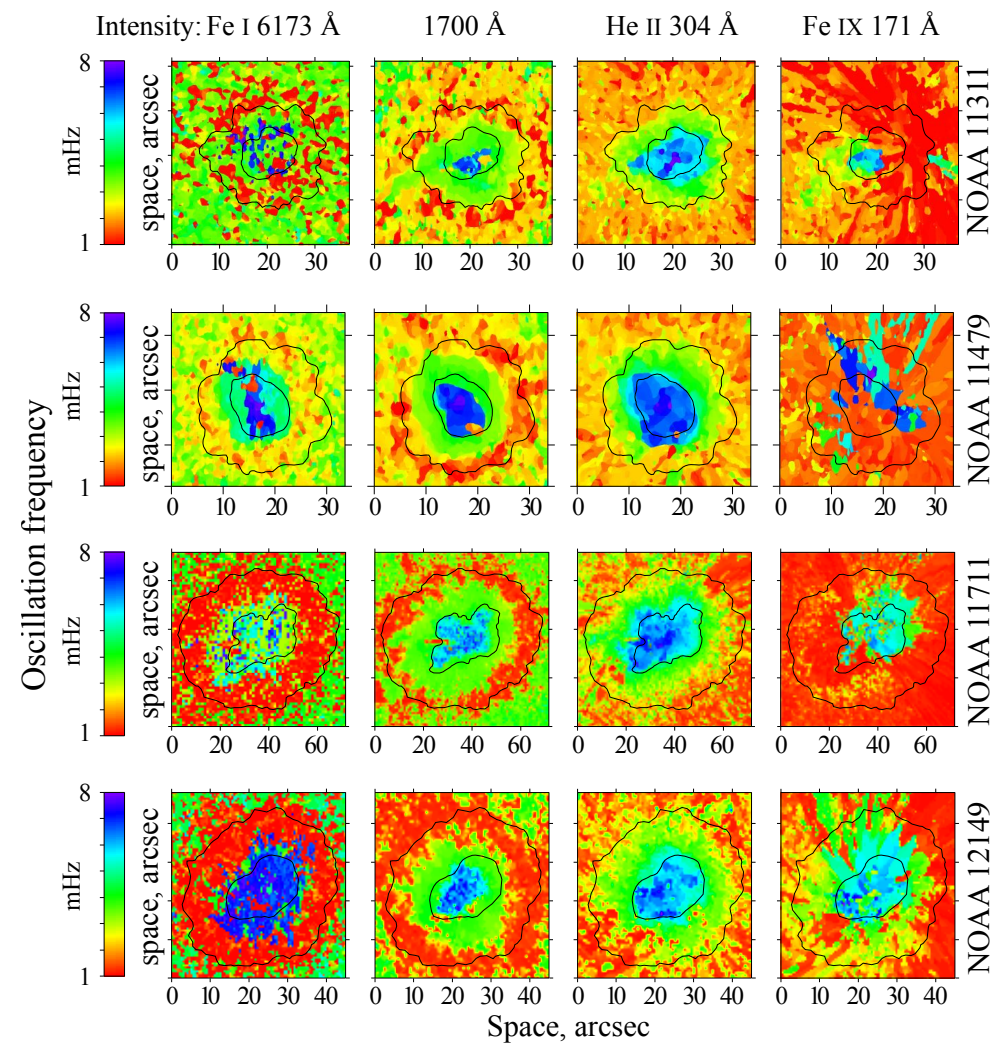

Figure 1. Dominant frequency distributions in sunspots at different height levels, from the photosphere to the corona. The black lines show the inner and outer penumbra boundaries as seen in the $1700 \AA$ band AIA images 


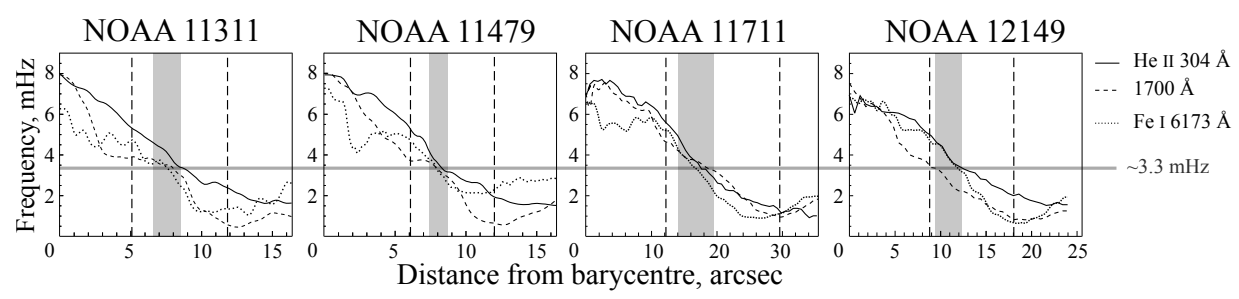

Figure 2. Radial distributions of the dominant frequencies at three heights in sunspots as functions of the distance from the barycentre. The vertical dashed lines mark the penumbra boundaries. The region of interest is marked with grey area, which corresponds to the averaging over the circular-shaped domain in the penumbra

(He II $304 \AA$ ) the highest peaks are shifted to the higher frequencies. This can be explained by the inhomogeneity of the penumbra or by the fact that sunspot has not purely circular shape. There are five-minute oscillations in the narrow region of the penumbra that dominate at all the heights (Figure 3). We consider this region to be a channel transporting five-minute oscillations from the photosphere up through the chromosphere.

The interesting behaviour in this penumbra region motivated a more detailed study of the distributions of several parameters there, including those found in earlier works by other researchers.

Figure 4 shows the azimuthally averaged magnetic field inclination at the He II $304 \AA$ line formation level estimated from the dominant frequency distributions. The details of the estimation procedure can be found in Kobanov, Kolobov, and Chelpanov (2015). The inner penumbra in these distributions is characterized by the steepest inclination angle gradient; the inclination angle there being $60-65^{\circ}$.

Various signatures indicating peculiar properties of the inner penumbrae of sunspots have been found previously by other authors. Identical field strength was found at three photospheric heights (deep photosphere, log $\tau_{500}=0$; mid photosphere, $\log \tau_{500}=-1.5$; and top of the photosphere, $\log \tau_{500}=-3$ ) by Borrero and Ichimoto (2011). At about the same distance from the sunspot centre, the magnetic field profiles of the three photospheric levels show the same value, and in the outer penumbra the order of the profiles is reversed (Figure 5). Bellot Rubio, Schlichenmaier, and Tritschler (2006), see Figure 3 therein, noted a hump in the inner penumbra in the azimuthally averaged temperatures at all the photospheric heights 


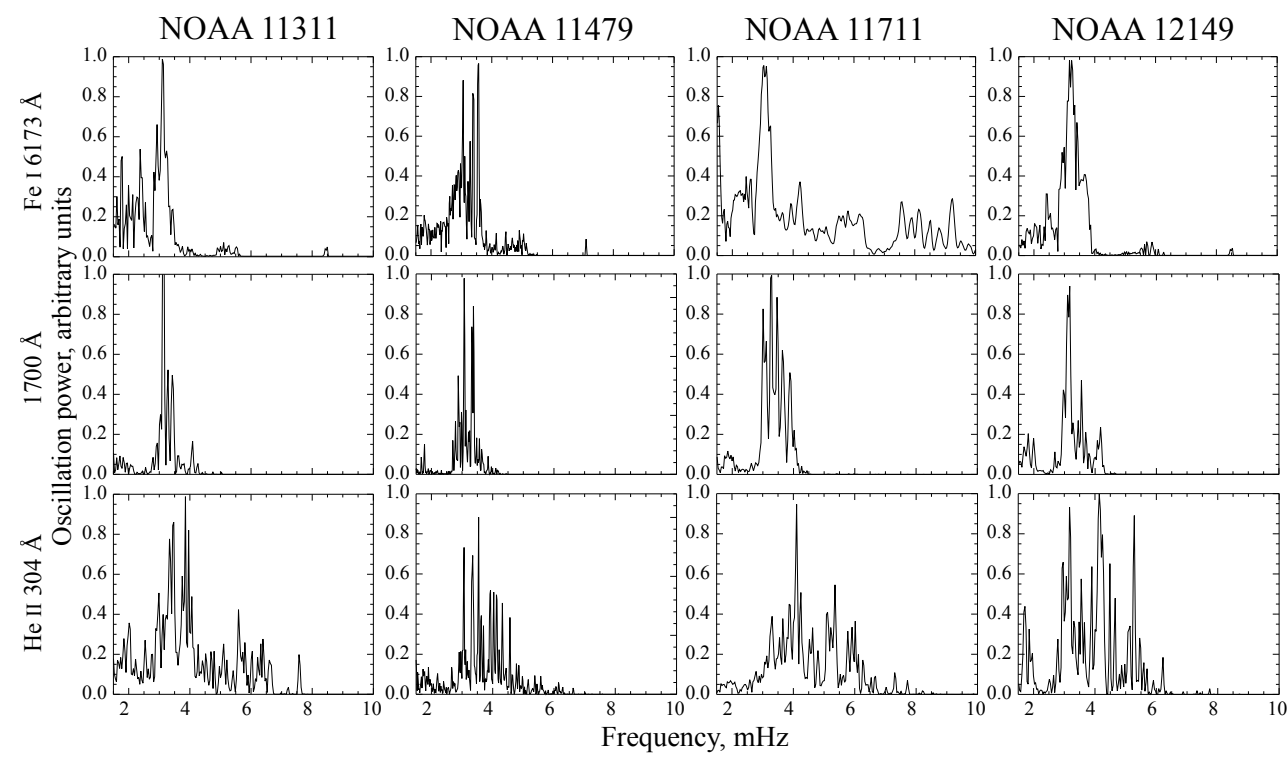

Figure 3. Spectra azimuthally averaged over the region within the penumbrae marked grey in Figure 2

$\left(-3 \leq \tau_{500} \leq 0\right)$ The amplitude of the hump was found to decrease with height. The authors suggested that these temperature enhancements could be due to hot penumbral tubes, by which plasma emerges from the subphotospheric layers. Based on these two works, one can conclude that the unique properties of the inner penumbrae seem to originate in deeper levels than that we study here.

These tubes are probably related to the photospheric and chromospheric Evershed flow, which peaks at the outer penumbra boundary and sharply terminates at the same region of the inner penumbra (see Figure 8 in Bellot Rubio, Schlichenmaier, and Tritschler (2006), and Figure 4 in Georgakilas et al. (2003)). Also, Bellot Rubio, Schlichenmaier, and Tritschler (2006) showed that the microturbulence velocity rapidly drops to zero in the inner penumbra.

Today, we lack a comprehensive explanation for this phenomenon. Probably, the key for understanding such a behaviour of the distributions is a model that describes penumbra magnetic field as a series of two types of interlocking-comb filaments (Weiss et al., 2004). The first one having a large inclination angle are located at low heights and dive beneath the photosphere 


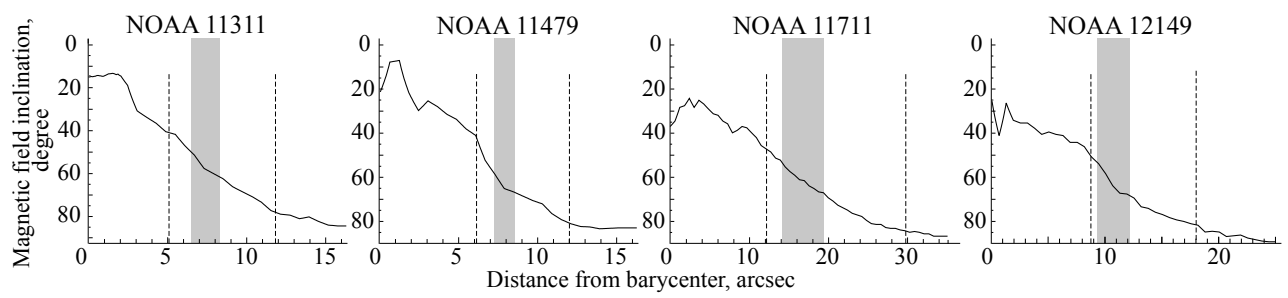

Figure 4. Estimation of the magnetic field inclination at the He II $304 \AA$ line height based on the dominant frequency distributions. The region of interest is marked with grey area

at the outer penumbra boundary. These filaments are associated with the Evershed flow. The second filament type is closer to vertical in orientation. Their magnetic field lines rise high in the atmosphere and either return to the surface far from the spot or form an open field line.

As follows from the aforesaid, interesting peculiarities are observed in the behaviour of a number of physical parameters in the sunspots' inner penumbra. In this paper, we hope to draw attention to this problem, solving which requires widening the height range of data analysis and modelling.

Acknowledgements. The study was performed with partial support of the Project No. 16.3.2 of ISTP SB RAS, by the Russian Foundation for Basic Research under grants No. 15-32-20504 mol_a_ved and 16-32-00268 mol_a. We acknowledge the NASA/SDO science team for providing the data. We are grateful to an anonymous referee for the helpful remarks and suggestions. 


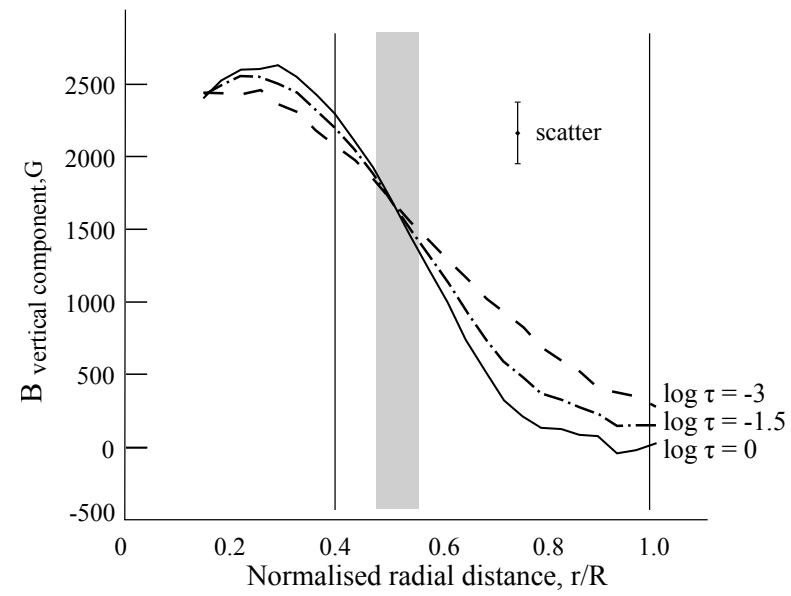

Figure 5. Magnetic field vertical component averaged over azimuth in sunspot NOAA 10923 in the deep photosphere at the continuum level, in the midphotosphere, and in the upper photosphere. The vertical lines mark the penumbra boundaries. The region of interest is marked with grey area (courtesy of Juan M. Borrero, see (Borrero and Ichimoto, 2011)) 


\section{References}

Alissandrakis, C.E., Georgakilas, A.A., Dialetis, D.: 1992, Dynamic phenomena in the chromospheric layer of a sunspot. Solar Phys. 138, 93105. DOI. ADS.

Beckers, J.M., Tallant, P.E.: 1969, Chromospheric Inhomogeneities in Sunspot Umbrae. Solar Phys. 7, 351-365. DOI. ADS.

Bellot Rubio, L.R.: 2003, The Fine Structure of the Penumbra: from Observations to Realistic Physical Models. In: Trujillo-Bueno, J., Sanchez Almeida, J. (eds.) Solar Polarization, Astronomical Society of the Pacific Conference Series 307, 301. ADS.

Bellot Rubio, L.R., Schlichenmaier, R., Tritschler, A.: 2006, Twodimensional spectroscopy of a sunspot. III. Thermal and kinematic structure of the penumbra at 0.5 arcsec resolution. Astron. Astrophys. 453, $1117-1127$. DOI. ADS.

Bloomfield, D.S., Lagg, A., Solanki, S.K.: 2007, The Nature of Running Penumbral Waves Revealed. Astrophys. J. 671, 1005-1012. DOI. ADS.

Bogdan, T.J., Judge, P.G.: 2006, Observational aspects of sunspot oscillations. Philosophical Transactions of the Royal Society of London Series A 364, 313-331. DOI. ADS.

Borrero, J.M., Ichimoto, K.: 2011, Magnetic Structure of Sunspots. Living Reviews in Solar Physics 8. DOI. ADS.

Botha, G.J.J., Arber, T.D., Nakariakov, V.M., Zhugzhda, Y.D.: 2011, Chromospheric Resonances above Sunspot Umbrae. Astrophys. J. 728, 84. DOI. ADS.

Brisken, W.F., Zirin, H.: 1997, New Data and Models of Running Penumbral Waves in Sunspots. Astrophys. J. 478, 814-816. ADS.

Cho, K.-S., Bong, S.-C., Nakariakov, V.M., Lim, E.-K., Park, Y.-D., Chae, J.C., Yang, H.-S., Park, H.-M., Yurchyshyn, V.: 2015, Intensity and Doppler Velocity Oscillations in Pore Atmospheres. Astrophys. J. 802, 45. DOI. ADS. 
Georgakilas, A.A., Christopoulou, E.B., Koutchmy, S.: 2000, Oscillations and running waves observed in sunspots. II. Photospheric waves. Astron. Astrophys. 363, 306-310. ADS.

Georgakilas, A.A., Christopoulou, E.B., Skodras, A., Koutchmy, S.: 2003, Chromospheric Evershed flow. Astron. Astrophys. 403, 1123-1133. DOI. ADS.

Giovanelli, R.G.: 1972, Oscillations and Waves in a Sunspot. Solar Phys. 27, $71-79$. DOI. ADS.

Jess, D.B., Reznikova, V.E., Van Doorsselaere, T., Keys, P.H., Mackay, D.H.: 2013, The Influence of the Magnetic Field on Running Penumbral Waves in the Solar Chromosphere. Astrophys. J. 779, 168. DOI. ADS.

Jess, D.B., Morton, R.J., Verth, G., Fedun, V., Grant, S.D.T., Giagkiozis, I.: 2015, Multiwavelength Studies of MHD Waves in the Solar Chromosphere. An Overview of Recent Results. Space Sci. Rev. 190, 103-161. DOI. ADS.

Khomenko, E., Collados, M.: 2015, Oscillations and Waves in Sunspots. Living Reviews in Solar Physics 12. DOI. ADS.

Kobanov, N., Kolobov, D., Chelpanov, A.: 2015, Oscillations Above Sunspots and Faculae: Height Stratification and Relation to Coronal Fan Structure. Solar Phys. 290, 363-380. DOI. ADS.

Kobanov, N.I.: 1990, On spatial characteristics of five-minute oscillations in the sunspot umbra. Solar Phys. 125, 25-30. DOI. ADS.

Kobanov, N.I., Makarchik, D.V.: 2004, Pulsating Evershed Flows and Propagating Waves in a Sunspot. Astronomy Reports 48, 954-964. DOI. ADS.

Kobanov, N.I., Chelpanov, A.A., Kolobov, D.Y.: 2013, Oscillations above sunspots from the temperature minimum to the corona. Astron. Astrophys. 554, A146. DOI. ADS.

Kobanov, N.I., Kolobov, D.Y., Chupin, S.A.: 2008, Traveling waves in the sunspot chromosphere: Problems and puzzles of experiments. Astronomy Letters 34, 133-140. DOI. ADS. 
Kobanov, N.I., Kolobov, D.Y., Makarchik, D.V.: 2006, Umbral Three-Minute Oscillations and Running Penumbral Waves. Solar Phys. 238, 231-244. DOI. ADS.

Kolobov, D.Y., Kobanov, N.I., Chelpanov, A.A., Kochanov, A.A., Anfinogentov, S.A., Chupin, S.A., Myshyakov, I.I., Tomin, V.E.: 2015, Behaviour of oscillations in loop structures above active regions. Advances in Space Research 56, 2760-2768. DOI. ADS.

Langhans, K., Scharmer, G.B., Kiselman, D., Löfdahl, M.G., Berger, T.E.: 2005, Inclination of magnetic fields and flows in sunspot penumbrae. Astron. Astrophys. 436, 1087-1101. DOI. ADS.

Lemen, J.R., Title, A.M., Akin, D.J., Boerner, C. P. F. and. Chou, Drake, J.F., Duncan, D.W., Edwards, F.M. C. G. and. Friedlaender, Heyman, G.F., Hurlburt, N.L. N. E. and. Katz, Kushner, G.D., Levay, M., Lindgren, D.P. R. W. and. Mathur, McFeaters, E.L., Mitchell, R.A. S. and. Rehse, Schrijver, C.J., Springer, R.A. L. A. and. Stern, Tarbell, T.D., Wuelser, C.J. J.-P. and. Wolfson, Yanari, C., Bookbinder, P.N. J. A. and. Cheimets, Caldwell, D., Deluca, R. E. E. and. Gates, Golub, L., Park, S., Podgorski, R.I. W. A. and. Bush, Scherrer, P.H., Gummin, P. M. A. and. Smith, Auker, G., Jerram, P., Pool, R. P. and. Soufli, Windt, D.L., Beardsley, S., Clapp, J. M. and. Lang, Waltham, N.: 2012, The Atmospheric Imaging Assembly (AIA) on the Solar Dynamics Observatory (SDO). Solar Phys. 275, $17-40$. DOI. ADS.

Lites, B.W.: 1988, Photoelectric observations of chromospheric sunspot oscillations. V - Penumbral oscillations. Astrophys. J. 334, 1054-1065. DOI. ADS.

Löhner-Böttcher, J., Bello González, N.: 2015, Signatures of running penumbral waves in sunspot photospheres. Astron. Astrophys. 580, A53. DOI. ADS.

Madsen, C.A., Tian, H., DeLuca, E.E.: 2015, Observations of Umbral Flashes and Running Sunspot Waves with the Interface Region Imaging Spectrograph. Astrophys. J. 800, 129. DOI. ADS.

Musman, S., Nye, A.H., Thomas, J.H.: 1976, Observations of penumbral waves in the photosphere. Astrophys. J. Lett. 206, L175-L178. DOI. ADS. 
Reznikova, V.E., Shibasaki, K.: 2012, Spatial Structure of Sunspot Oscillations Observed with SDO/AIA. Astrophys. J. 756, 35. DOI. ADS.

Reznikova, V.E., Shibasaki, K., Sych, R.A., Nakariakov, V.M.: 2012, Three-minute Oscillations above Sunspot Umbra Observed with the Solar Dynamics Observatory/Atmospheric Imaging Assembly and Nobeyama Radioheliograph. Astrophys. J. 746, 119. DOI. ADS.

Rouppe van der Voort, L.H.M., Rutten, R.J., Sütterlin, P., Sloover, P.J., Krijger, J.M.: 2003, La Palma observations of umbral flashes. Astron. Astrophys. 403, 277-285. DOI. ADS.

Scherrer, P.H., Schou, J., Bush, R.I., Kosovichev, A.G., Bogart, R.S., Hoeksema, J.T., Liu, Y., Duvall, T.L., Zhao, J., Title, A.M., Schrijver, C.J., Tarbell, T.D., Tomczyk, S.: 2012, The Helioseismic and Magnetic Imager (HMI) Investigation for the Solar Dynamics Observatory (SDO). Solar Phys. 275, 207-227. DOI. ADS.

Sigwarth, M., Mattig, W.: 1997, Velocity and intensity oscillations in sunspot penumbrae. Astron. Astrophys. 324, 743-749. ADS.

Solanki, S.K., Montavon, C.A.P.: 1993, Uncombed fields as the source of the broad-band circular polarization of sunspots. Astron. Astrophys. 275, 283. ADS.

Sych, R.: 2015, MHD waves in sunspots. ArXiv e-prints. ADS.

Sych, R., Nakariakov, V.M.: 2014, Wave dynamics in a sunspot umbra. Astron. Astrophys. 569, A72. DOI. ADS.

Title, A.M., Frank, Z.A., Shine, R.A., Tarbell, T.D., Topka, K.P., Scharmer, G., Schmidt, W.: 1993, On the magnetic and velocity field geometry of simple sunspots. Astrophys. J. 403, 780-796. DOI. ADS.

Tsiropoula, G., Alissandrakis, C.E., Mein, P.: 2000, Association of chromospheric sunspot umbral oscillations and running penumbral waves. I. Morphological study. Astron. Astrophys. 355, 375-380. ADS.

Tziotziou, K., Tsiropoula, G., Mein, N., Mein, P.: 2004, On the Nature of Chromospheric Umbral Flashes And Running Penumbral Waves (Abstract). In: Laskarides, P. (ed.) Hellenic Astronomical Society Sixth Astronomical Conference, 50. ADS. 
Tziotziou, K., Tsiropoula, G., Mein, N., Mein, P.: 2006, Observational characteristics and association of umbral oscillations and running penumbral waves. Astron. Astrophys. 456, 689-695. DOI. ADS.

Weiss, N.O., Thomas, J.H., Brummell, N.H., Tobias, S.M.: 2004, The Origin of Penumbral Structure in Sunspots: Downward Pumping of Magnetic Flux. Astrophys. J. 600, 1073-1090. DOI. ADS.

Yuan, D., Sych, R., Reznikova, V.E., Nakariakov, V.M.: 2014, Multi-height observations of magnetoacoustic cut-off frequency in a sunspot atmosphere. Astron. Astrophys. 561, A19. DOI. ADS.

Zirin, H., Stein, A.: 1972, Observations of Running Penumbral Waves. Astrophys. J. Lett. 178, L85. DOI. ADS. 